\title{
Recommended respiratory tests are not routinely performed for mucopolysaccharidosis patients
}

\begin{abstract}
To the Editor:
Copyright @The authors 2022

This version is distributed under the terms of the Creative Commons Attribution NonCommercial Licence 4.0. For commercial reproduction rights and permissions contact permissions@ersnet.org

Received: 27 May 2021 Accepted: 21 Dec 2021

\section{○ @®@}

Mucopolysaccharidoses (MPS) are a group of rare genetic lysosomal storage disorders. The seven types of MPS exhibit a wide spectrum of clinical severity, including cognitive impairment, skeletal and joint abnormalities, short stature, coarsened facial features, vision loss, and cardiovascular and respiratory impairment, which are the leading causes of morbidity and mortality among MPS patients [1-3]. Ear, nose and throat (ENT) and upper airway manifestations are initially more pronounced, and may lead to the initial diagnosis. Lower airway disease becomes more frequent with increasing age. Symptoms can be tracheomalacia, stenosis and bronchomalacia. Bronchitis and pneumonia can appear, and are due to increased secretions and poor airway clearance. Restrictive lung disease predominates among patients with significant skeletal involvement, namely MPS-IV and MPS-VI. Sleep disturbances and obstructive sleep apnoea secondary to airway obstruction are common [4]. Specific therapy, such as enzyme replacement therapy (ERT), is available for most types of MPS.

Monitoring respiratory function is therefore crucial. French guidelines for MPS management, which are based on several international guidelines, were published in July 2016. Depending on the type of MPS, the guidelines recommend performing the following examinations: 1) spirometry every 1-3 years, or sooner if there are respiratory symptoms; 2) a 6-min walk test (6MWT) every 12 months; 3) a sleep disorder study, mainly by polysomnography (PSG), every 1-3 years, or more frequently if clinically indicated; 4) measurement of blood gases every 1-3 years or if there are respiratory symptoms; or 5) a chest radiograph once a year if the patient has a central catheter or shows respiratory symptoms $[1,5-8]$. We conducted a retrospective study to evaluate the feasibility of the recommended respiratory tests and their informativeness for MPS children and adolescents, and to explore the likely reasons for not performing them.

We collected data from the medical records of each patient for the three previous years (July 2016 to July 2019). The results of the last evaluation were recorded. The number of expected tests was calculated based on French guidelines depending on the type of MPS, age and respiratory symptoms for each patient.

There is no age limit in the French guidelines for performing examinations. Based on the literature, we considered that spirometry was achievable for patients aged $\geqslant 6$ years. Patients $<4$ years of age were considered too young for the 6MWT. Reasons for failure were collected from the medical records. If this information was not available, clinicians in charge of the patients were asked for their opinion. The study was approved by the local Ethics Committee on Human Research (Tours, France).

49 MPS children (21 with MPS-I, 10 with MPS-II, nine with MPS-III, six with MPS-IV, two with MPS-VI and one with MPS-VII) under the age of 18 years (median age 8.4 years, range 1.5-18.0 years) from five French centres specialised in the management of inborn errors of metabolism were enrolled between July and December 2019. 44 patients (90\%, 95\% CI 81-98\%) showed respiratory symptoms at inclusion. 19 tests were not performed because patients were too young (9\%, 95\% CI 5-13\%). Excluding these tests, only $43 \%$ (95\% CI 36-50\%) of the recommended respiratory tests were performed (84 interpretable tests conducted of the 195 recommended). Five tests were attempted but not interpretable (two spirometries, two 6MWTs and one PSG) and were classified as failed.

\section{Shareable abstract (@ERSpublications)}

Recommended respiratory tests used as major outcomes in clinical trials for MPS treatment cannot be routinely performed in everyday practice because neurocognitive impairment and motor skill difficulties affect compliance for most MPS patients https://bit.ly/3G4qp8U

Cite this article as: Denamur S, Touati G, Debelleix S, et al. Recommended respiratory tests are not routinely performed for mucopolysaccharidosis patients. ERJ Open Res 2022; 8: 00567-2021 [DOI: 10.1183/23120541.00567-2021].
\end{abstract}


The test achievement rate for each test and the main reason for failure for unconducted tests are presented in figure 1. More precisely, spirometry was performed on patients with more severe skeletal and joint abnormalities $(\mathrm{p}<0.01)$, such as MPS-IV or -VI patients, whereas they were never performed on the 10 MPS-II patients from our series $(p<0.01)$. All patients for whom PSG was performed had chronic ENT symptoms and most were MPS-I (13 out of 17 patients; 76\%, 95\% CI 56-97\%). The major reason for failure for the 6MWT, spirometry and PSG was, in more than half of the cases, neurocognitive impairment, which negatively affected understanding of the instructions and compliance. Motor skill impairment was also a contributing factor for failing to perform the 6MWT. By contrast, the main reason for not measuring blood gases or performing a chest radiograph was that these two examinations were generally considered to be poorly informative by practitioners. There were no significant differences between medical centres in the test achievement rate, except for the achievement rate of measuring blood gases, which was higher in Tours than at the other centres (Chi-square test $\mathrm{p}<0.001$ ), and that of performing a chest radiograph, which was higher in Tours and Toulouse than in Angers and Bordeaux (Chi-square test $\mathrm{p}<0.001$ ). There were no significant differences in the main reason for failing to conduct each test.

Finally, this observational and multicentre study showed that recommended respiratory evaluations were not performed in everyday practice. The major reasons for such low adherence were neurological impairment and poor comprehension, motor impairment, or the physician's opinion that such tests do not provide useful information. Tests proposed in French and international recommendations for the follow-up of MPS have been repeatedly used as major outcomes to assess the efficacy of specific treatment, especially ERT. For example, phase I/II and phase III extension trials of ERT with laronidase demonstrated safety and clinical benefits for MPS-I, with a significant improvement in forced vital capacity, walking distance during the 6MWT and detection of obstructive sleep apnoea [9, 10]. Similarly, the approval of idursulfase for MPS-II was based on data from a clinical trial that demonstrated that ERT significantly improved the distance covered during the 6MWT and forced vital capacity [11]. In these studies, the results for spirometry and the 6MWT were available for most of the patients, contrasting with the results of our observational study. A major difference was that most of the patients enrolled in these trials had an attenuated phenotype, contrasting with the high prevalence of neurological involvement of patients in our study cohort. For example, in the initial trials designed to evaluate the safety and efficacy of ERT in MPS-I, all patients enrolled had attenuated disease, with no or moderate cognitive impairment $[9,10]$. The ability to perform reproducible spirometry and to walk a minimum distance in 6MWT were part of the inclusion criteria. In our cohort, a greater number of test results were available for MPS-IV patients, a disease not known to have central nervous system involvement, which could explain the better compliance to perform these tests [7, 12]. On the contrary, blood gas measurements and chest radiographs are easy to perform, and do not require patient cooperation. However, these two examinations were not often performed because their results were considered to be generally uninformative. In our cohort, the results of blood gas measurements were always normal and chest radiographs never revealed a previously unknown condition.
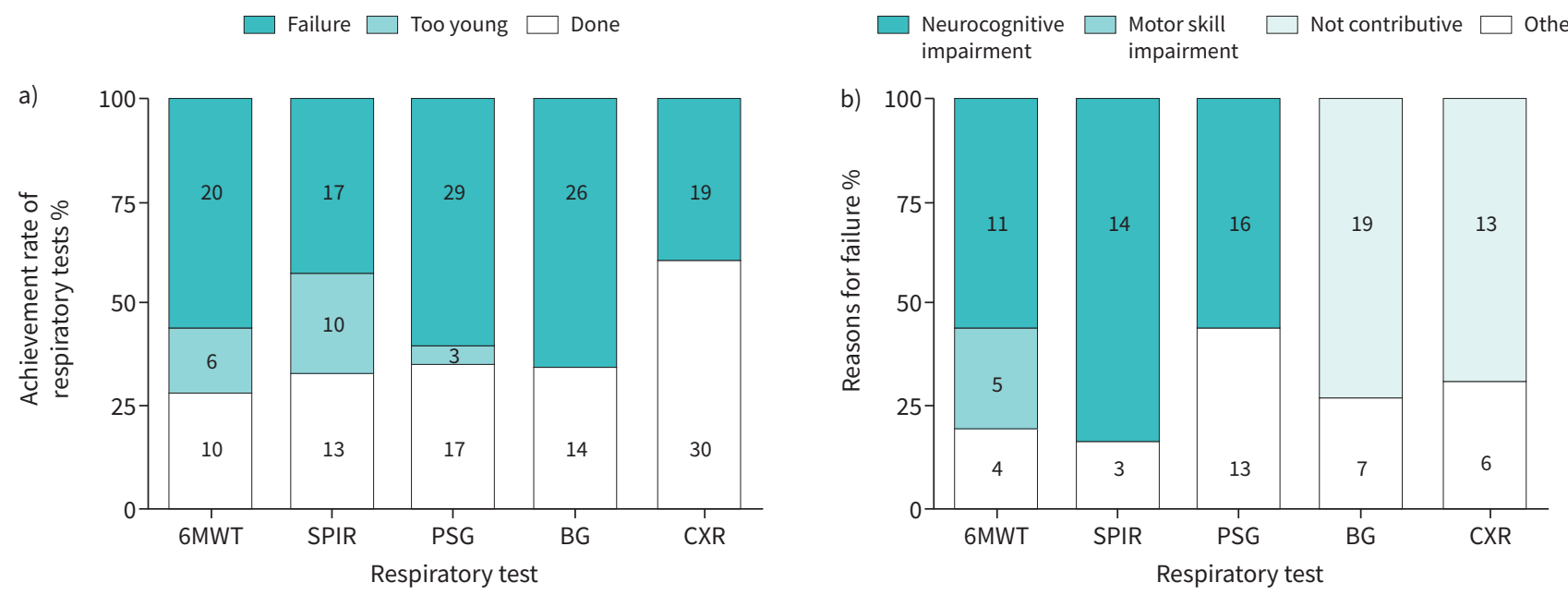

FIGURE 1 a) Achievement rate and b) reasons for failure of recommended respiratory tests for 49 mucopolysaccharidosis patients. Results are expressed as the percentage of patients. The absolute number of patients in each group is specified on the corresponding bar. 6MWT: 6-min walk test; SPIR: spirometry; PSG: polysomnography; BG: blood gas analysis; CXR: chest radiography. 
There is a need to develop new tools to evaluate pulmonary function in MPS patients. Airway resistance measurement by body plethysmography requires a patient's cooperation. Therefore, it does not appear to be well adapted for the youngest children or those with neurological impairment. Interrupter technique resistance measurements or an impulse oscillometry system could be alternatives to spirometry for young patients or those with cognitive impairment MPS. Indeed, these tests do not require the forced expiration technique but rather only a few normal breaths, allowing children as young as 2-3 years old to be tested. However, airway resistance measurements may not provide information about a potential restrictive defect. The measurement of functional residual capacity by helium dilution could evaluate a useful volume and requires less cooperation than plethysmography. Night-time oximetry, which requires less cooperation and provides an evaluation of obstructive sleep apnoea, could be an alternative to PSG.

Among imaging examinations, pulmonary magnetic resonance imaging (MRI) has the advantage of being nonionising, and can evaluate pulmonary volumes and, consequently, pulmonary function. However, this examinaiton is longer than a chest computed tomography scan and MRI requires cooperation from the child, which is difficult to obtain from young children or those with severe neurological impairment without sedation. In any case, French guidelines recommend medullar and cerebral MRI in the follow-up of neurological disease for MPS patients. These examinations are generally performed under sedation. In such cases, pulmonary sequences could be added to medullar and cerebral sequences. However, no study has yet been published on pulmonary MRI in MPS patients. Previous evaluations and measurements in healthy children appear to be essential to secondarily interpret MPS MRI images.

In summary, our results highlight the difficulties of assessing the respiratory involvement of MPS patients and the necessity to develop new tools. They also raise the question of how to confirm treatment efficacy for more severe MPS patients, as improvement has only been demonstrated for patients with attenuated phenotypes.

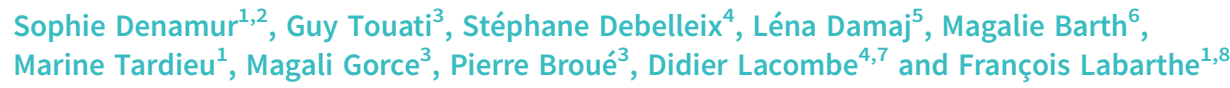

${ }^{1}$ Centre de Référence Maladies Métaboliques ToTeM, CHRU Tours, Tours, France. ${ }^{2}$ Centre d'Etude des Pathologies Respiratoires, Inserm UMR1100, Université François Rabelais de Tours, Tours, France. ${ }^{3}$ Centre de Référence Maladies Métaboliques, CHU Toulouse, Toulouse, France. ${ }^{4}$ Pneumologie Pédiatrique et Service de Médecine du sommeil, and CIC Pédiatrique CIC-P Bordeaux 1401, CHU Bordeaux, Bordeaux, France. ${ }^{5}$ Centre de Compétences Maladies Métaboliques, CHU Rennes, Rennes, France. ${ }^{6}$ Centre de Compétences Maladies Métaboliques, CHU Angers, Angers, France. ${ }^{7}$ Rare Diseases Genetics and Metabolism, Inserm U1211, Université de Bordeaux, Bordeaux, France. ${ }^{8} \mathrm{~N} 2 \mathrm{C}$, Inserm U1069, Université François Rabelais de Tours, Tours, France.

Corresponding author: François Labarthe (labarthe@univ-tours.fr)

Provenance: Submitted article, peer reviewed.

Conflict of interest: D. Lacombe reports personal fees from Sanofi-Genzyme outside the submitted work. The other authors all declare no conflict of interest.

References

1 Stapleton M, Hoshina H, Sawamoto K, et al. Critical review of current MPS guidelines and management. Mol Genet Metab 2019; 126: 238-245.

2 Burton BK, Jego V, Mikl J, et al. Survival in idursulfase-treated and untreated patients with mucopolysaccharidosis type II: data from the Hunter Outcome Survey (HOS). J Inherit Metab Dis 2017; 40: 867-874.

3 Lavery C, Hendriksz CJ, Jones SA. Mortality in patients with Sanfilippo syndrome. Orphanet J Rare Dis 2017; 12: 168.

4 Nashed A, Al-Saleh S, Gibbons J, et al. Sleep-related breathing in children with mucopolysaccharidosis. J Inherit Metab Dis 2009; 32: 544-550.

5 HAS. Protocole National de Diagnostic et de Soins (PNDS) Mucopolysaccharidoses n.d. Saint-Denis, Haute Authorité de Santé, 2016.

6 Akyol MU, Alden TD, Amartino $\mathrm{H}$, et al. Recommendations for the management of MPS VI: systematic evidence- and consensus-based guidance. Orphanet J Rare Dis 2019; 14: 118. 
7 Hendriksz CJ, Berger KI, Giugliani R, et al. International guidelines for the management and treatment of Morquio A syndrome. Am J Med Genet A 2015; 167A: 11-25.

8 Martins AM, Dualibi AP, Norato D, et al. Guidelines for the management of mucopolysaccharidosis type I. J Pediatr 2009; 155: S32-S46.

9 Clarke LA, Wraith JE, Beck M, et al. Long-term efficacy and safety of laronidase in the treatment of mucopolysaccharidosis I. Pediatrics 2009; 123: 229-240.

10 Wraith JE. Mucopolysaccharidoses and mucolipidoses. Handb Clin Neurol 2013; 113: 1723-1729.

11 Muenzer J, Wraith JE, Beck M, et al. A phase II/III clinical study of enzyme replacement therapy with idursulfase in mucopolysaccharidosis II (Hunter syndrome). Genet Med 2006; 8: 465-473.

12 Harmatz PR, Mengel KE, Giugliani R, et al. Longitudinal analysis of endurance and respiratory function from a natural history study of Morquio A syndrome. Mol Genet Metab 2015; 114: 186-194. 